\title{
TRATAMENTO CIRÚRGICO DA FRATURA INSTÁVEL DO ANEL PÉLVICO EM PACIENTES ESQUELETICAMENTE IMATUROS
}

\author{
SURGICAL TREATMENT OF UNSTABLE PELVIC \\ RING FRACTURE IN SKELETALLY IMMATURE PATIENTS
}

\begin{abstract}
João Antonio Matheus Guimarães', Ricardo de Souza Portes Meirelles², Luiz Augusto Peçanha Tavares Júnior², Flávio Goldszłajn ${ }^{3}$, Tito Rocha ${ }^{3}$, Pedro Henrique Barros Mendes ${ }^{4}$
\end{abstract}

\section{RESUMO}

Objetivo: Apresentar o resultado do tratamento cirúrgico definitivo da fratura instável do anel pélvico na criança submetida a redução e estabilização cirúrgica. Métodos: Avaliamos 10 pacientes com esqueleto imaturo que sofreram fraturas instáveis do anel pélvico tratados cirurgicamente no período entre março de 2004 a janeiro de 2008. O estudo foi retrospectivo, baseado na avaliação clínica e radiográfica. Resultados: A média etária na época do trauma foi de 8,8 anos (dois a 13 anos), sendo sete do sexo feminino e três do sexo masculino. As causas dos traumas foram atropelamento em oito casos, e acidente com motocicleta e queda de altura em um caso cada. Cinco pacientes apresentavam lesões associadas, como fraturas da clavícula, diáfise do fêmur, úmero proximal, ossos da perna, olecrânio e lesão de bexiga. Todos os pacientes avaliados apresentaram excelente evolução clínica. A assimetria pélvica antes do procedimento cirúrgico variava de 0,7 a 2,9cm (média $1,45 \mathrm{~cm}$ ), e caiu para valores entre 0,2 a $0,9 \mathrm{~cm}$ (média $0,39 \mathrm{~cm}$ ) após a redução. Em nenhum dos casos houve alteração da assimetria pélvica medida no pós-operatório imediato e no fim do seguimento. Conclusão: A fratura do anel pélvico em pacientes esqueleticamente imaturos é rara e a indicação de tratamento cirúrgico não é usual. Diversos autores questionam o tratamento conservador devido às complicações encontradas. A remodelação óssea não parece suficiente para que ocorra uma melhora da assimetria pélvica, fator que justifica a opção pelo tratamento cirúrgico para a redução e correção das deformidades do anel pélvico.

Descritores - Fixação de Fratura; Criança; Pelve

\section{ABSTRACT}

Objectives: To present the outcome of the definitive surgical treatment of unstable fracture of the pelvic ring in children submitted to surgical reduction and stabilization. Methods: We studied 10 patients with immature skeletons that suffered unstable fractures of the pelvic ring treated during the period between March 2004 and January 2008. The study was retrospective, based on clinical and radiographic evaluations. Results: The mean age at the time of trauma was 8.8 years ( 2 to 13 years). Seven patients were female and three male. The cause of the trauma was being run over in eight cases, and one case each of a motorcycle accident and falling from a height. Five patients had other associated injuries such as fractures of the clavicle, femur diaphysis, proximal humerus, fibula, olecranon, and bladder injury. All patients showed an excellent clinical outcome. The pelvic asymmetry before surgery ranged from 0.7 to $2.9 \mathrm{~cm}$ (mean $1.45 \mathrm{~cm}$ ), and dropped to values between 0.2 and $0.9 \mathrm{~cm}$ (mean $0.39 \mathrm{~cm}$ ) after reduction. In no cases was a change observed in pelvic asymmetry measured in the immediate postoperative period and at the end of follow-up. Conclusion: Pelvic ring fractures in skeletally immature patients is rare and surgical treatment is not usual. Several authors have questioned conservative treatment due to the complications encountered. Bone remodeling is not enough to improve pelvic asymmetry, a factor that justifies the choice of surgical treatment for the reduction and correction of pelvic ring deformities.

Keywords - Fracture Fixation; Child; Pelvis

\footnotetext{
1 - Chefe do Serviço de Ortopedia e Traumatologia do Instituto Nacional de Traumatologia e Ortopedia (INTO/MS).

2 - Médico Residente do Instituto Nacional de Traumatologia e Ortopedia (INTO/MS).

3 - Médico do Centro de Cirurgia de Trauma Ortopédico do Instituto Nacional de Traumatologia e Ortopedia (INTO/MS).

4 - Chefe do Centro de Cirurgia da Criança e Adolescente do Instituto Nacional de Traumatologia e Ortopedia (INTO/MS).
}

Trabalho realizado no Instituto Nacional de Traumatologia e Ortopedia do Ministério da Saúde (INTO/MS).

Correspondência: Av. Ataulfo de Paiva, 135/1.117 - Leblon - 22440-030 - Rio de Janeiro, RJ. E-mail: jomatheus@uol.com.br

Trabalho recebido para publicação: 27/10/09, aceito para publicação: 26/04/10. 


\section{INTRODUÇÃO}

As fraturas do anel pélvico em pacientes esqueleticamente imaturos são relativamente raras, representam menos de $0,2 \%$ de todas as fraturas pediátricas e 1 a 5\% das admissões em centros terciários de trauma infantil $^{(1,2)}$. Geralmente causadas por trauma de alta energia é frequente a associação a outras lesões orgânicas (aparelho geniturinário, vísceras abdominais, sistema nervoso central, estruturas neurovasculares e musculoesqueléticas) $)^{(3)}$.

Apesar de sua relativa baixa incidência, lesões pélvicas em crianças representam grande morbidade e mortalidade ${ }^{(4)}$. Um estudo postmortem, que avaliou 66 mortes causadas por trauma em crianças, revelou fratura pélvica e hemorragia severa em $42 \%$ dos casos como causa da morte. Assim como nos adultos, a hemorragia retroperitoneal é a pior complicação nas fraturas instáveis da pelve ${ }^{(5-7)}$.

Comparados aos adultos, as crianças com fratura do anel pélvico sobrevivem mais às lesões associadas, com isso representam um custo socioeconômico e morbidade a longo prazo maiores. Em um estudo prospectivo realizado com 95 crianças vítimas de fraturas do anel pélvico, avaliou-se a capacidade de locomoção e dependência desses pacientes. $\mathrm{O}$ resultado mostrou que 80\% dos pacientes com fraturas instáveis, e 52\% dos com fraturas estáveis, apresentavam-se dependentes de ajuda para locomoção ${ }^{(7)}$.

O tratamento conservador das fraturas do anel pélvico em crianças através de repouso, tração esquelética e aparelho gessado tem sido preconizado devido ao potencial de correção da lesão pela remodelação óssea que ocorre nesta faixa etária. Entretanto, muitos casos evoluem desfavoravelmente, devido a potenciais complicações, caracterizadas por assimetria pélvica, escoliose não estrutural, lombalgia, dismetria dos membros inferiores e claudicação durante a marcha. Algumas lesões em que existam desvios importantes, especialmente a presença de luxação sacroilíaca devido a uma instabilidade vertical, ou mesmo em casos de instabilidade horizontal acentuada, o tratamento conservador não resulta em função satisfatória. A presença da cartilagem trirradiada aberta na criança favorece a remodelação, porém a literatura ainda não apresenta consenso quanto a melhor forma de tratamento destas graves lesões, e muita controvérsia persiste sobre quais crianças podem se beneficiar com o tratamento invasivo ${ }^{(1,8)}$. Não há dúvida que, na emergência, nos casos com instabilidade hemodinâmica, de maneira similar ao adulto, o tratamento imediato deve ser através da utilização do fixador externo, visando controlar o sangramento e salvar a vida do paciente, mesmo que de maneira temporária. Em casos com disjunção da articulação sacroilíaca, apenas o fixador externo anterior não será suficiente para reduzir e estabilizar o anel posterior ${ }^{(4,5,9)}$.

O propósito deste estudo é apresentar o resultado do tratamento cirúrgico definitivo da fratura instável do anel pélvico na criança, submetida a redução e estabilização cirúrgica em caráter eletivo, após o atendimento inicial efetuado em um serviço de emergência.

\section{MÉTODOS}

Após aprovação do comitê de ética em pesquisa, avaliamos 10 pacientes com esqueleto imaturo que sofreram fraturas instáveis do anel pélvico tratados cirurgicamente no Instituto Nacional de Traumatologia e Ortopedia do Ministério da Saúde (INTO/MS) no período entre março de 2004 e janeiro de 2008. Os pacientes selecionados foram submetidos a avaliação clínica ambulatorial e análise de radiografias pré e pós-operatórias. Todos os pacientes receberam o primeiro atendimento em outro serviço de emergência, sendo referenciados para a nossa instituição devido à complexidade da lesão, para o tratamento definitivo. O critério de inclusão dos pacientes neste estudo foi a lesão instável do anel pélvico, em paciente com sinais radiográficos de esqueleto imaturo, definido pela presença da cartilagem trirradiada do ilíaco. Os pacientes com lesão estável ou com padrão de fratura sem desvio, seja da fratura do ilíaco ou do sacro, foram excluídos desta casuística.

A técnica cirúrgica empregada baseou-se no princípio da redução e estabilização das partes anterior e posterior do anel pélvico. O componente anterior, seja a fratura dos ramos ou a disjunção da sínfise pubiana, foi reduzido de maneira indireta através da colocação de uma montagem com fixador externo tubular, tipo AO, composta de apenas dois pinos de Schanz na região supra-acetabular de cada ilíaco, com o auxílio da radioscopia, através das incidências em obturatriz e alar do ilíaco. O diâmetro dos pinos de Schanz utilizados foram de acordo com o tamanho do osso do paciente, sendo que em três casos o diâmetro era de 5,0mm; nos demais, pinos de $3,5 \mathrm{~mm}$ devido às dimensões da pelve em questão, com as correspondentes conexões e barras para a montagem anterior do fixador externo. Em apenas um caso, existiu necessidade de realizarmos um 
acesso anterior a sínfise pubiana para a redução direta da luxação, devido ao tempo de evolução da lesão, e por este motivo uma placa de $1 / 3$ de cano de $3,5 \mathrm{~mm}$ foi utilizada na fixação óssea. Quanto à lesão posterior do anel, a técnica da redução indireta através da manipulação anterior com os pinos do fixador externo, associado a tração longitudinal do membro inferior, possibilitou a utilização de um parafuso canulado colocado de maneira percutânea na articulação sacroilíaca lesada. Em seis casos o diâmetro deste parafuso foi de 4,0mm, devido ao corredor de segurança para a sua colocação ser menor pelas dimensões do osso imaturo. Em três casos o parafuso canulado apresentava diâmetro de 7,0mm, pois eram crianças maiores em que o tamanho da pelve suportava esta dimensão. Em apenas um caso, em que a lesão posterior era uma fratura "em crescente" da asa do ilíaco, a fixação anterior com a montagem supraacetabular do fixador externo foi utilizada isoladamente, com consolidação óssea ocorrendo sem perda de redução obtida. A montagem do fixador externo anterior foi retirada entre seis e oito semanas, após a comprovação radiológica de consolidação das fraturas. Em todos os nove casos, em que o parafuso canulado foi utilizado para a estabilização posterior da articulação sacroilíaca, não foi realizada a retirada deste implante.

O estudo foi retrospectivo, baseado na avaliação dos prontuários médicos e da avaliação clínica e radiográfica dos pacientes com um acompanhamento médio de 808,83 dias do trauma inicial. O desfecho do estudo baseou-se na quantificação radiográfica da assimetria pélvica residual após o tratamento cirúrgico, com a utilização do chamado método de Keshishyan et $a l^{(5)}$ (Figura 1), comparando-se os achados pré e pós operatórios. Por este método, a assimetria pélvica é determinada pela diferença, em centímetros, entre duas linhas diagonais traçadas a partir da borda da articulação sacroilíaca até a cartilagem trirradiada contralateral. A variação anatômica considerada normal desta medição é de 4mm de assimetria, que pode ocorrer devido à rotação da pelve no momento da radiografia, porém acima de $5 \mathrm{~mm}$ esta alteração deve ser considerada como anormal ${ }^{(6)}$. A assimetria pélvica era também correlacionada com o índice de deformidade, que é a diferença entre as duas diagonais divididas pela soma das mesmas, para assegurar um erro mínimo devido a esta rotação da bacia na radiografia em anteroposterior.

Os pacientes foram analisados clinicamente através de entrevista e exame físico. Avaliamos o arco de movimento e força abdutora da articulação do quadril,

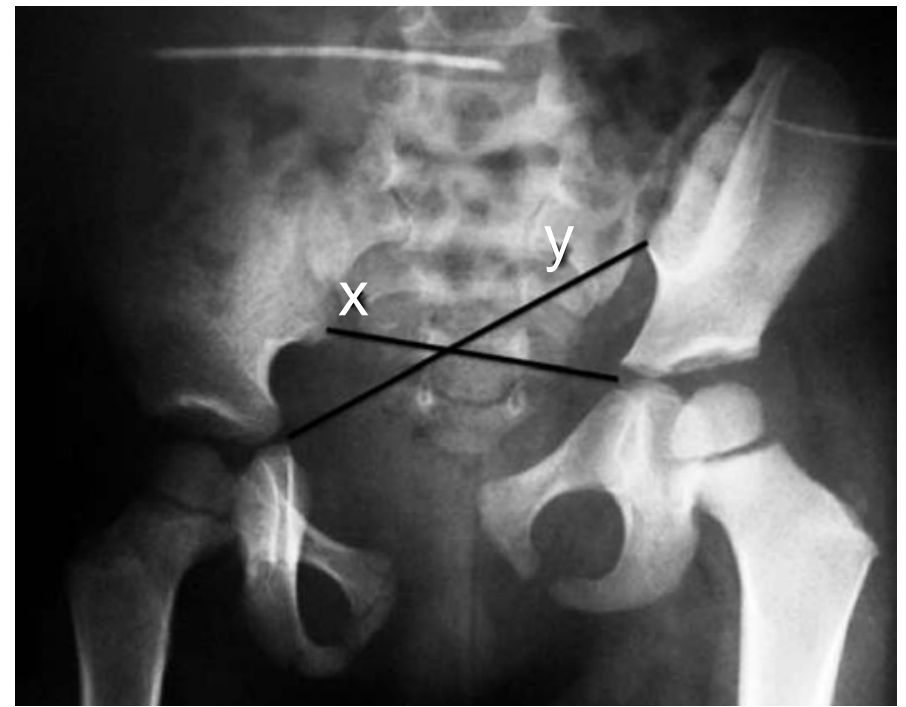

Figura 1 - Método de mensuração da obliquidade pélvica de Keshishyan et $a^{\left({ }^{(5)}\right.}$, em que a assimetria pélvica é a diferença entre as diagonais $(\mathrm{X}-\mathrm{Ycm})$. $\mathrm{O}$ índice de deformidade corresponde a diferença das diagonais $(X-Y c m)$ dividido pela soma das diagonais $(X+Y c m)$.

desvios da coluna vertebral, sequela neurológica, característica da marcha e a existência de dor referida ou à palpação da pelve. Estes dados eram correlacionados com os achados radiográficos do paciente.

\section{RESULTADOS}

Dos pacientes avaliados, sete eram do sexo feminino e três do masculino. A média etária na época do trauma foi de 8,8 anos (dois a 13 anos). Os pacientes foram operados com uma média de 11,9 dias de fratura (variou de quatro a 29 dias). A causa do trauma inicial foi atropelamento em oito casos, e acidente com motocicleta e queda de altura um caso cada. As lesões osteoarticulares encontradas no anel pélvico foram divididas em anteriores e posteriores, sendo a disjunção da sínfise pubiana presente em cinco pacientes, fratura de dois ramos em três e fratura dos quatro ramos em dois. As lesões posteriores corresponderam em seis casos à luxação unilateral da articulação sacroilíaca, em um existia uma fratura unilateral do ilíaco posterior, em um abertura anterior unilateral da articulação sacroilíaca, em um existia luxação da sacroilíaca de um lado e compressão da outra decorrente de compressão lateral e em um de luxação bilateral das articulações sacroilíacas. O mecanismo do trauma foi considerado como compressão lateral em três casos, compressão anteroposterior em dois e cisalhamento vertical em cinco. A classificação AO-OTA ${ }^{(10)}$ foi utilizada para avaliação dos pacientes com a seguinte distribuição: 61 B1 (um caso); 61 B2 (um caso); 61 
C1 (seis casos); 61 C2 (um caso) e 61 C3 (um caso) (Tabela 1). Todos os pacientes receberam atendimento inicial em outra unidade de saúde, sendo transferidos para o nosso hospital após a estabilização clínica inicial. Cinco pacientes apresentavam outras lesões associadas, sendo um caso com fratura da clavícula que foi tratado sem cirurgia com imobilização externa. Um paciente apresentava fratura diafisária de fêmur, tratado com hastes intramedulares flexíveis. Um paciente apresentava fratura do úmero proximal e fraturas dos ossos da perna ipsilaterais à lesão da pelve, sendo o membro superior tratado conservadoramente e a tíbia fixada com hastes intramedulares flexíveis. Uma fratura do olecrânio da paciente que sofreu queda de altura, foi submetida à osteossíntese com banda de tensão. Um paciente apresentou lesão concomitante de bexiga, diagnosticada através de laparotomia exploradora, no atendimento inicial, que evoluiu durante a internação com infecção urinária.

A principal complicação do método foi a presença de três casos de infecção superficial no trajeto dos pinos de Schanz da montagem anterior do fixador externo, as quais regrediram totalmente após a retirada dos mesmos. Os pacientes ficaram em média 40,5 dias (variação de 28 a 48 dias) com fixador externo. O tempo de internação variou de dois a 14 dias, média de 3,8 dias. Todos os pacientes avaliados apresentaram excelente evolução clínica, o que foi constatado durante as consultas de controle. Nenhum caso evoluiu com dor na pelve, alteração na marcha, discrepância de membros inferiores ou escoliose ao exame físico. Não foi detectada lesão vascular ou neurológica decorrente da utilização do parafuso canulado na articulação sacroilíaca.

Quanto à avaliação radiográfica, a assimetria pélvica antes do procedimento cirúrgico variou de 0,7 a 2,9cm (média $1,45 \mathrm{~cm}$ ), e caiu para valores entre 0,2 a $0,9 \mathrm{~cm}$ (média 0,39cm) após a redução. O tempo de evolução mínimo foi de 210 dias, máximo 1.896 dias (Tabela 2). Em nenhum caso houve alteração da assimetria pélvica medida no pós-operatório imediato e no fim do seguimento, mostrando não ter ocorrido remodelação óssea suficiente para alterar esta medida, durante o período estudado (Figura 2).

Tabela 1 - Dados clínicos dos pacientes.

\begin{tabular}{|c|c|c|c|c|c|c|c|c|}
\hline Caso & $\begin{array}{l}\text { Idade } \\
\text { (anos) }\end{array}$ & Sexo & $\begin{array}{c}\text { Classificação } \\
\text { AO-ОTA }\end{array}$ & $\begin{array}{c}\text { Mecanismo de } \\
\text { trauma }\end{array}$ & Lesão posterior & $\begin{array}{l}\text { Lesão } \\
\text { anterior }\end{array}$ & $\begin{array}{l}\text { Causa do } \\
\text { acidente }\end{array}$ & Lesões associadas \\
\hline \multirow[t]{2}{*}{1} & 2 & Fem & $61 \mathrm{C} 1$ & Cisalhamento & Luxação & Disjunção & Atropelamento & Nenhuma \\
\hline & & & & vertical & sacroilíaca & Sinfise & & \\
\hline \multirow[t]{2}{*}{2} & 13 & Fem & $61 \mathrm{~B} 1$ & Compressão & Abertura & Fratura & Acidente de moto & Nenhuma \\
\hline & & & & anteroposterior & anterior sacrollíaca & quatro ramos & & \\
\hline \multirow[t]{2}{*}{3} & 8 & Fem & $61 \mathrm{~B} 2$ & Compressão & Luxação & Fratura & Atropelamento & Nenhuma \\
\hline & & & & lateral & sacroilíaca & dois ramos & & \\
\hline \multirow[t]{2}{*}{4} & 4 & Fem & $61 \mathrm{C} 1$ & Cisalhamento & Luxação & Disjunção & Atropelamento & Fratura de \\
\hline & & & & vertical & sacroilíaca & Sínfise & & fêmur \\
\hline \multirow[t]{2}{*}{5} & 7 & Masc & $61 \mathrm{C} 1$ & Cisalhamento & Luxação & Disjunção & Atropelamento & Fratura de \\
\hline & & & & vertical & sacroilíaca & Sínfise & & clavícula \\
\hline \multirow[t]{2}{*}{6} & 6 & Fem & $61 \mathrm{C} 1$ & Cisalhamento & Luxação & Disjunção & Atropelamento & Nenhuma \\
\hline & & & & vertical & sacroilíaca & Sínfise & & \\
\hline \multirow[t]{2}{*}{7} & 13 & Masc & $61 \mathrm{C} 2$ & Compressão & Luxação & Fratura & Atropelamento & Lesão de \\
\hline & & & & lateral & $\begin{array}{l}\text { sacroilíaca + compressão } \\
\text { anterior (bilateral) }\end{array}$ & dois ramos & & bexiga \\
\hline \multirow[t]{2}{*}{8} & 10 & Masc & $61 \mathrm{C} 1$ & Cisalhamento & Fratura ilíaco & Fratura & Atropelamento & Nenhuma \\
\hline & & & & vertical & posterior & dois ramos & & \\
\hline \multirow[t]{2}{*}{9} & 12 & Fem & $61 \mathrm{C} 3$ & Compressão & Luxação & Fratura & Atropelamento & Fratura de \\
\hline & & & & lateral & sacroilíaca(bilateral) & quatro ramos & & úmero + fratura de tíbia \\
\hline \multirow[t]{2}{*}{10} & 13 & Fem & $61 \mathrm{C} 1$ & Cisalhamento & Luxação & Disjunção & Queda altura & Fratura de \\
\hline & & & & vertical & sacroilíaca & Sínfise & & olecrânio \\
\hline
\end{tabular}


Tabela 2 - Resultados do tratamento instituído.

\begin{tabular}{|c|c|c|c|c|c|c|}
\hline Caso & $\begin{array}{l}\text { Tempo entre } \\
\text { o acidente e } \\
\text { cirurgia(dias) }\end{array}$ & Cirurgia realizada & $\begin{array}{c}\text { Assimetria } \\
\text { pélvicapré-op(cm) }\end{array}$ & $\begin{array}{c}\text { Assimetria } \\
\text { pélvicapré-op }(\mathrm{cm})\end{array}$ & $\begin{array}{c}\text { Índice de } \\
\text { deformidadepré-op } \\
\text { (Keshishyan ) }\end{array}$ & $\begin{array}{c}\text { Índice de } \\
\text { deformidadepós-op } \\
\text { (Keshishyan) }\end{array}$ \\
\hline \multirow[t]{3}{*}{1} & 16 & Placa sínfise & 1,7 & 0,2 & 0,2 & 0,02 \\
\hline & & + & & & & \\
\hline & & Parafuso SI & & & & \\
\hline \multirow[t]{3}{*}{2} & 5 & Fixador externo & 0,7 & 0,3 & 0,06 & 0,02 \\
\hline & & + & & & & \\
\hline & & Parafuso SI & & & & \\
\hline \multirow[t]{3}{*}{3} & 11 & Fixador externo & 1,8 & 0,3 & 0.17 & 0,04 \\
\hline & & + & & & & \\
\hline & & Parafuso SI & & & & \\
\hline \multirow[t]{3}{*}{4} & 6 & Fixador externo & 1,2 & 0,5 & 0,15 & 0,04 \\
\hline & & + & & & & \\
\hline & & Parafuso SI & & & & \\
\hline \multirow[t]{3}{*}{5} & 14 & Fixador externo & 1,2 & 0,2 & 0,09 & 0,03 \\
\hline & & + & & & & \\
\hline & & Parafuso SI & & & & \\
\hline \multirow[t]{3}{*}{6} & 14 & Fixador externo & 1,5 & 0,6 & 0,09 & 0,05 \\
\hline & & + & & & & \\
\hline & & Parafuso SI & & & & \\
\hline \multirow[t]{3}{*}{7} & 29 & Fixador externo & 2,9 & 0,9 & 0,15 & 0,09 \\
\hline & & + & & & & \\
\hline & & Parafuso SI & & & & \\
\hline 8 & 12 & Fixador externo & 0,8 & 0,2 & 0,12 & 0,01 \\
\hline \multirow[t]{3}{*}{9} & 8 & Fixador externo & 1,5 & 0,5 & 0,07 & 0,03 \\
\hline & & + & & & & \\
\hline & & Parafusos SI Bilateral & & & & \\
\hline \multirow[t]{3}{*}{10} & 4 & Fixador externo & 1,2 & 0,2 & 0,09 & 0,03 \\
\hline & & + & & & & \\
\hline & & Parafuso SI & & & & \\
\hline
\end{tabular}
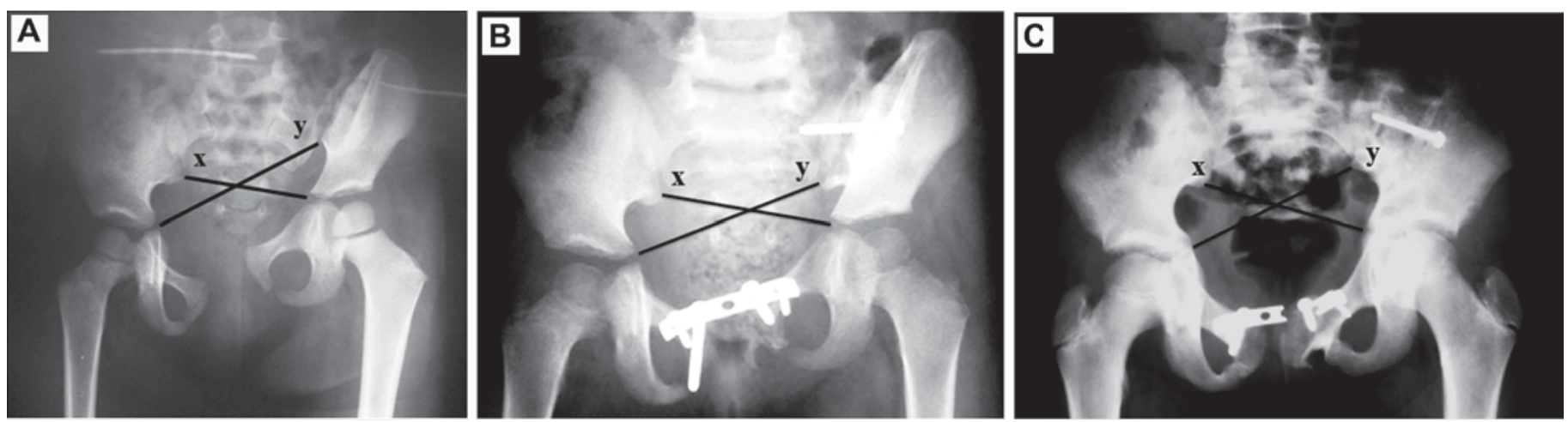

Figura 2 - Menina, dois anos de idade, apresentando luxação da articulação sacroilíaca à esquerda e disjunção da sínfise púbica. Radiografias panorâmicas de bacia em AP: A) Pré-operatório. Assimetria = 0,7cm; B) Pós-operatório imediato. Assimetria = 0,3cm; C) Após seis anos de evolução, demonstrando consolidação e ausência de alteração. 


\section{DISCUSSÃO}

Fraturas instáveis do anel pélvico em crianças são relativamente raras e decorrentes a trauma de alta energia. Em nossa casuística o atropelamento em via pública foi a principal causa. Assim como ocorre nas lesões do anel pélvico em adultos, vêm acompanhadas de outras lesões que apresentam alta morbidade e mortalidade ${ }^{(8,11)}$. Apesar disso, sabe-se pouco a respeito da evolução dessas lesões a longo prazo. O perfil das crianças estudadas é similar ao relatado na literatura, exceto pela distribuição quanto ao sexo. Apesar de serem lesões mais incidentes em meninos, dos 10 casos estudados, sete (70\%) eram do sexo feminino. A causa mais frequente responsável pelo acidente foi o atropelamento em via pública, o que está de acordo com o exposto por Signorino et $a l^{(4)}$ que teve como causas mais comuns os atropelamentos e acidentes automobilísticos, justificando o trauma de alta energia necessário para causar este dano na pelve da criança que, por apresentar um osso mais plástico do que o adulto, absorve mais a energia gerada no impacto inicial. Essa capacidade da criança se reflete também na menor mortalidade após um trauma de alta energia com lesão do anel pélvico, estatisticamente a lesão fatal ocorre em $5 \%$ dos casos em crianças e $17 \%$ em adultos $^{(9)}$.

Diversos estudos $^{(12-15)}$ analisaram a evolução a longo prazo de lesões instáveis e estáveis do anel pélvico tratadas conservadoramente. Schwarz et al ${ }^{(16)}$, relatam $47 \%$ de maus resultados devido à assimetria pélvica, e enfatiza a importância de se obter uma redução anatômica e conclui que fraturas instáveis do anel pélvico tratadas conservadoramente evoluem com resultado funcional inaceitável. Outros autores ${ }^{(3,13,17)}$ apresentam casos de fraturas instáveis do anel pélvico tratados não cirurgicamente que evoluíram com resultado insatisfatório, com dor residual na região posterior da bacia, discrepância de membros inferiores, sinal de Trendelenburg e escoliose lombar devido à assimetria pélvica residual. Nenhum dos casos tratados cirurgicamente de nossa casuística evoluiu com dor residual, alterações na marcha ou qualquer outra alteração ao exame físico. Nosso estudo apresenta como limitação a relativa pequena casuística, porém como se trata de uma fratura rara que acomete cerca de uma por 100.000 crianças por ano, acreditamos que a amostra é relevante, pois não existem grandes séries publicadas sobre o tratamento cirúrgico desta lesão. Os resultados nos estimulam a continuar indicando o tratamento cirúrgico para os casos em que exista uma assimetria pélvica acima de $0,5 \mathrm{~cm}$ e, principalmente, quando estiver associada a uma luxação da articulação sacroilíaca, a qual dificilmente por medidas não cirúrgicas evoluirá satisfatoriamente. A opção da associação da fixação posterior com o parafuso canulado sacroilíaco, baseia-se no fato de esta técnica ser pouco invasiva, propiciar uma estabilidade mecânica adequada na parte posterior do anel e se mostrar um método seguro e reprodutível na criança, pois a aplicação isolada do fixador externo anterior não consegue controlar os elementos posteriores do anel pélvico $^{(18)}$ (Figura 3). Smith et al. ${ }^{(1,3)}$, em sua casuística mostram que os pacientes tratados com redução e fixação anterior e posterior combinada tiveram uma assimetria pélvica residual menor que os pacientes tratados com a fixação externa anterior isolada.

O diâmetro do parafuso e dos pinos de Schanz da montagem anterior do fixador externo devem ser adequados às dimensões do osso da criança em questão, o parafuso canulado de 4,0mm mostrou-se suficiente para estabilizar pelves menores, em que o corredor de segurança da articulação sacroilíaca era pequeno para a
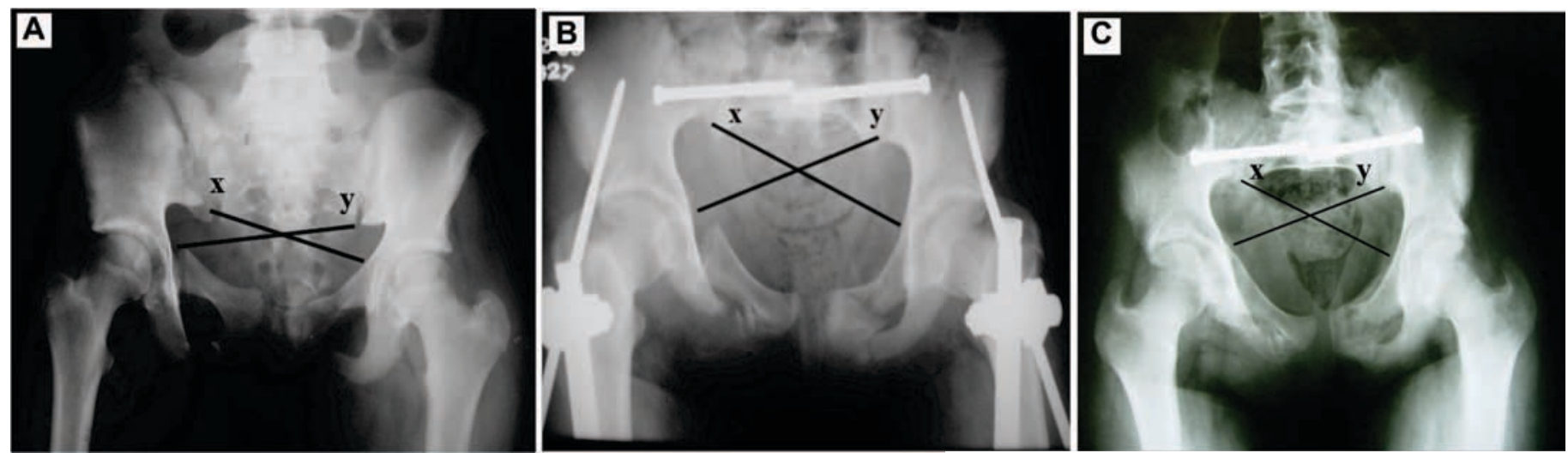

Figura 3 - Menina, 12 anos de idade, vítima de atropelamento, apresentando luxação da articulação sacroilíaca bilateral e fratura dos ramos púbicos. Radiografias panorâmicas de bacia em AP: A) Pré-operatório. Assimetria = 1,5cm; B) Pós-operatório imediato. Assimetria $=0,5 \mathrm{~cm} ;$ C) Após três meses de evolução. Assimetria $=0,5 \mathrm{~cm}$. 
utilização do diâmetro de 7,0mm. Da mesma forma, os pinos de Schanz com diâmetro de 3,5mm, permitiram a colocação da montagem anterior do fixador externo, nos casos em que as dimensões do ilíaco não permitia um diâmetro de pino maior na região supra-acetabular.

\section{CONCLUSÃO}

A fratura do anel pélvico em pacientes esqueleticamente imaturos é evento raro, e a indicação de tratamento cirúrgico não é usual. Diversos autores questionam o tratamento conservador, preconizado há vários anos para estas fraturas, devido às complicações encontradas, principalmente a discrepância de comprimento dos membros inferiores e a dor residual na articulação sacroilíaca. A remodelação óssea que normalmente ocorre na criança em crescimento não parece ser suficiente para que ocorra uma melhora da assimetria pélvica, fator este que justifica a opção do tratamento cirúrgico para a redução e correção das deformidades do anel pélvico, de maneira precoce, por ocasião da lesão.

\section{REFERÊNCIAS}

1. Smith WR, Oakley M, Morgan SJ. Pediatric pelvic fractures. J Pediatr Orthop. 2004;24(1):130-5.

2. Chia JPY, Holland AJ, Little D, Cass DT. Pelvic fractures and associated injuries in children. J Trauma. 2004;56(1):83-8.

3. Smith W, Shurnas P, Morgan S, Agudelo J, Luszko G, Knox EC, et al. Clinical outcomes of unstable pelvic fractures in skeletally immature patients. J Bone Joint Surg Am. 2005;87(11):2423-31.

4. Signorino PR, Densmore J, Werner M, Winthrop A, Stylianos S, Guice KS, et al. Pediatric pelvic injury: functional outcome at 6-month follow-up. J Pediatr Surg. 2005;40(1):107-13.

5. Keshishyan RA, Rozinov VM, Malakhov AO, Kuznetsov LE, Strunin EG, Chogovadze GA, et al. Pelvic polyfractures in children. Radiographic diagnosis and treatment. Clin Orthop Relat Res. 1995;(320):28-33.

6. Upperman JS, Gardner M, Gaines B, Schall L, Ford HR. Early functional outcome in children with pelvic fractures. J Pediatr Surg. 2000;35(6):1002-5.

7. Blasier DR, McAtee J, White R, Mitchell DT. Disruption of the pelvic ring in pediatric patients. Clin Orthop Relat Res. 2000;(376):87-95.

8. Demetriades D, Karaiskakis M, Velmahos GC, Alo K, Murray J, Chan L. Pelvic fractures in pediatric and adult trauma patients: are they different injuries? J Trauma. 2003;54(6):1146-51.

9. Cothren CC, Moore EE, Smith WR, Morgan SJ. Preperitoneal pelvic packing in the child with na unstable pelvis: a novel approach. J Pediatr Surg. 2006;41(4):E17-9. 10. Marsh JL, Slongo TF, Agel J, Broderick JS, Creevey W, DeCoster TA et al. Fracture and dislocation classification compendium - 2007. Orthopaedic Trauma Association classification, database and outcomes committee. J Orthop Trauma. 2007;21(Suppl 10):S1-133.

11. Ismail N, Bellemere JF, Mollitt DL, DiScala C, Koeppel B, Tepas JJ 3rd. Death from pelvic fracture: children are different. J Pediatr Surg. 1996;31(1):82-5.

12. Heeg M, Klasen $\mathrm{HJ}$. Long-term outcome of sacroiliac disruption in children. J Pediatr Orthop. 1997;17(3):337-41.

13. McDonald GA. Pelvic disruptions in children. Clin Orthop Relat Res. 1980;(151):130-4.

14. Bryan WJ, Tullos HS. Pediatric pelvic fractures: review of 52 patients. J Trauma. 1979;19(11):799-805.

15. Rieger H, Brug E. Fractures of the pelvis in children. Clin Orthop Relat Res. 1997;(336):226-39.

16. Schwarz N, Posch E, Mayr J, Fischmeister FM, Scwarz AF, Öhner T. Long-term results of unstable pelvic ring fractures in children. Injury. 1998;29:431-433.

17. Quick TJ, Eastwood DM. Pediatric fractures and dislocations of the hip and pelvis. Clin Orthop Rel Res. 2005;(432):87-96.

18. Matta JM, Anderson LM, Epstein HC, Hendrick P. Fractures of the acetabulum. A retrospective analysis. Clin Orthop Relat Res. 1986;(205):230-40. 